\title{
Functions of mammalian SIRT4 in cellular metabolism and research progress in human cancer (Review)
}

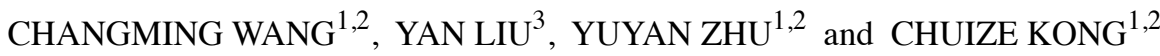 \\ ${ }^{1}$ Department of Urology, The First Affiliated Hospital of China Medical University; \\ ${ }^{2}$ Department of Urological Laboratory, The First Affiliated Hospital of China Medical University, Shenyang, Liaoning 110001; \\ ${ }^{3}$ Department of Pharmaceutical Toxicology, School of Pharmacy, China Medical University, Shenyang, \\ Liaoning 110122, P.R. China
}

Received November 1, 2019; Accepted May 18, 2020

DOI: $10.3892 / \mathrm{ol} .2020 .11872$

\begin{abstract}
Sirtuins are mammalian homologs of yeast silent information regulator two (SIRT) and are a highly conserved family of proteins, which act as nicotinamide adenine dinucleotide $\left(\mathrm{NAD}^{+}\right)$-dependent histone deacetylases. The seven sirtuins (SIRT1-7) share a conserved catalytic core domain; however, they have different enzyme activities, biological functions, and subcellular localizations. Among them, mitochondrial SIRT4 possesses ADP-ribosyltransferase, $\mathrm{NAD}^{+}$-dependent deacetylase, lipoamidase, and long-chain deacylase activities and can modulate the function of substrate proteins via ADP-ribosylation, delipoylation, deacetylation and long-chain deacylation. SIRT4 has been shown to play a crucial role in insulin secretion, fatty acid oxidation, amino acid metabolism, ATP homeostasis, apoptosis, neurodegeneration, and cardiovascular diseases. In addition, recent studies have demonstrated that SIRT4 acts as a tumor suppressor. Here, the present review summarizes the enzymatic activities and biological functions of SIRT4, as well as its roles in cellular metabolism and human cancer, which are described in the current literature.
\end{abstract}

\section{Contents}

1. Introduction

2. Structural characteristics of the sirtuin family

3. Enzymatic activities, substrates, and cellular functions of SIRT4

4. Functions of SIRT4 in human cancer

5. Conclusion

Correspondence to: Professor Chuize Kong, Department of Urology, The First Affiliated Hospital of China Medical University, 155 Nanjingbei Street, Shenyang, Liaoning 110001, P.R. China E-mail: kongchuize_cmu@sina.cn

Key words: SIRT4, molecular structure, enzyme activities, cellular metabolism, cancer

\section{Introduction}

The acylation of proteins is one of the modifications, which occurs following translation and can modulate the function of the proteins by changing the surface charge and regulating protein conformations or protein-protein interactions, which is similar to phosphorylation (1). Histone deacetylases (HDACs) can remove the acetylated group from the $\mathrm{N}$-acetyl-lysine of histones or non-histones. To date, four types of histone deacetylases have been discovered, class I include HDAC1-3 and HDAC8, class II include HDAC4-7 and HDAC9-10, and HDAC11 belongs to class IV $(1,2)$. Sirtuins are defined as class III HDACs and are dependent on $\mathrm{NAD}^{+}$, which distinguishes them from the classes I, II and IV, which are zinc-dependent (3). In the 1970s, the silent information regulator 2 gene (SIRT) was first discovered in budding yeast, as a regulator of chromatin structure (4). This group of proteins are now known to be also distributed in a wide range of mammal species, such as drosophila, murine and cattle $(4,5)$. In humans, the SIRT family consists of seven members, SIRT1-7, which are divided into four classes with respect to their sequence information. SIRT-1, -2 , and -3 are class I proteins, SIRT4 is class II, SIRT5 is class III, and SIRT- 6 and -7 are class IV $(6,7)$. All of the sirtuins share a highly conserved NAD ${ }^{+}$-binding catalytic core composed of 270 amino acids but vary in their $\mathrm{N}$ - and $\mathrm{C}$-terminal sequences. The different $\mathrm{N}$ - and $\mathrm{C}$-terminal sequences are important for subcellular localization and enzyme activities $(8,9)$.

These seven mammalian sirtuins have different protein structures, subcellular localizations, functional properties, enzymatic activities, and substrate specificities (Table I) (10). SIRT-1, -6 , and -7 are primarily located in the nucleus, SIRT2 is primarily located in the cytoplasm, and SIRT3-5 are predominately found in the mitochondria (11). However, some of these proteins are known to translocate from their primary location in different tissues or under various physiological conditions (12). For example, in myoblast cell line, SIRT1 moves to the cytosol under the action of some kinases and PI3K signal cascade (8). and SIRT2 moves into the nucleus during the $\mathrm{G}_{2} / \mathrm{M}$ cell cycle transition (13). Each sirtuin also has unique catalytic activities, which enable the sirtuins to exhibit broad and important regulatory functions in numerous 
biological processes, such as life span, gene transcription, cell proliferation, differentiation, apoptosis, genome stability, cellular metabolism, tumorigenesis, energy homeostasis, DNA damage, and stress responses $(7,14-17)$.

In contrast to other sirtuins, research on mitochondrial SIRT4 is relatively limited. However, it is well-known that SIRT4 is widely expressed in human organs and tissues, particularly in the heart, liver, kidney, spleen, prostate, testis, and ovaries (18). Unlike other sirtuins, SIRT4 possesses a conserved deacetylase domain, but earlier reports suggested it lacked detectable histone deacetylase activity (19). In 2006, Haigis et al (20) first discovered that SIRT4 possesses ADP-ribosyltransferase activity. Subsequently, it was found to have substrate-specific deacetylase, lipoamidase, and long-chain deacylase activities (21). These catalytic activities provide SIRT4 with the ability to play a vital role in insulin secretion, fatty oxidation, leucine catabolism, ATP homeostasis, lipid catabolism, tumorigenesis, neurological disorders and cardiovascular diseases $(7,21)$. However, SIRT4 is still the least well-known sirtuin, as these activities are weak. It has been suggested that SIRT4 will become a novel therapeutic target and molecules which modulate its activity will be developed to treat various diseases in the future $(22,23)$. In the present review, SIRT4 will be discussed and its functions in cellular metabolism and human cancer.

\section{Structural characteristics of the sirtuin family}

As aforementioned, every member of the sirtuin family contains a highly conserved catalytic center comprised of $\sim 270$ amino acids and divergent $\mathrm{N}$ - or $\mathrm{C}$-terminal sequences $(24,25)$. Therefore, the structure of sirtuins was divided into the non-enzymatic and the enzymatic parts. The non-enzymatic part includes seven specific $\mathrm{N}$ - and $\mathrm{C}$-terminal segments, which determines the subcellular localization and catalytic function of the sirtuin (25). The presence of an N-terminal mitochondrial targeting sequence ensures that mitochondrial SIRT3-5 localize within the mitochondrial matrix. When these signal sequences are cleaved, the enzymatic functions of these proteins are activated $(26,27)$.

The enzymatic part of the seven sirtuins is characterized by a high degree of fidelity. In 2001, Finnin et al (28) first identified the structure of sirtuins, and SIRT2 was the first reported subtype, which almost represents the structural basis of all sirtuins in the enzymatic part. The catalytic core consists of two main parts: A conserved large Rossmann fold domain and a variable small domain (28). The large inverted Rossmann fold domain consists of $6 \beta$-strands and $6 \alpha$-helices. The small domain contains a zinc finger module and a helical module (29). These two modules of the small domain are joined to the large Rossmann fold domain by four polypeptide chains, which form a large groove between the small and large domains. This junctional groove includes the $\mathrm{NAD}^{+}$-binding site, and most of the residues are highly conserved. The catalytic core of sirtuins are also included in the junctional groove, and mutations within these sites result in the loss of deacetylation catalytic activity (28,30-33). Moreover, when the substrate is close to the center of SIRTs, a conformational rearrangement occurs, and the small domain is rotated by $\sim 25$, thereby changing the conformation of the binding site to allow the catalytic reaction to proceed in a continuous process $(34,35)$.

With respect to SIRT4, the non-enzymatic part has no C-terminal sequence and only contains 28 positively charged amino acids in the N-terminal sequence, which serves as a mitochondrial localization signal with low sequence conservation (36,37). In the enzymatic part, the typical sirtuin structure is located, which consists of a large Rossmann fold domain with a conserved His 160 catalytic residue and a small domain. There are 2 specific structures, which separate SIRT4 from the other isoforms. The first one is a flexible loop, which contains an additional 12 residues (residues 195-206) in the $\mathrm{Zn}^{2+}$-binding module. This loop is located deep within the catalytic core, which contributes to substrate binding and restrains relevant active site dynamics. The second unusual structure is the channel in the catalytic core, which starts from the acyl-Lys binding tunnel and terminates at the protein surface. This channel has a small positively charged area on the outer entrance and is predominantly hydrophobic. This channel serves as the binding site for longer substrate acyls and regulatory metabolites $(37,38)$. The positive potential of the channel accounts for its weak $\mathrm{NAD}^{+}$-dependent deacetylase, which is within the 14-600 $\mu \mathrm{M}$ range, and also explains why the positively charged NADH was easier to combine with SIRT4 and the mitochondrial concentration of free NADH, $\mathrm{NAD}^{+} / \mathrm{NADH}$ ratio could act as a physiological SIRT4 regulator $(39,40)$.

\section{Enzymatic activities, substrates, and cellular functions of SIRT4}

The robust enzymatic activities of SIRT4 are not fully understood; however, SIRT4 is associated with numerous cellular metabolic processes, such as insulin secretion, glutamine metabolism, and fatty acid metabolism (21). The principal enzymatic activities of SIRT4, involved in these biological processes, are ADP-ribosyltransferase, $\mathrm{NAD}^{+}$-dependent deacetylase, lipoamidase, and long-chain deacylase (Table II). These functions will be described in more detail and are also summarized in Fig. 1.

ADP-ribosyltransferase and insulin secretion. Glutamine is the most abundant free cytoplasmic amino acid and has been found to be the staple nitrogen donor for the synthesis of nucleotides and amino acids (41). It can be converted into glutamate and $\alpha$-ketoglutarate $(\alpha-\mathrm{KG})$ by two consecutive reactions and subsequently enters the TCA cycle. Glutamine is first converted into glutamate by glutaminase, then it is further converted into the TCA cycle intermediate $\alpha$-KG either by glutamate dehydrogenase (GDH) or transamination-coupled reactions $(42,43)$. GDH was the first mitochondrial protein to be identified to be inactivated through mono-ADP-ribosylation, which was found to be catalyzed by SIRT4. SIRT4 directly and enzymatically transfers the ADP-ribosyl group from $\mathrm{NAD}^{+}$to the $\mathrm{C} 172$ histone residue of GDH, thereby inhibiting its function and ultimately inhibiting the metabolism of glutamine in the mitochondria and reducing ATP production $(20,42,44)$.

The inhibitory effect of SIRT4 on GDH represses insulin secretion in response to glucose and amino acids in pancreatic $\beta$ cells. As insulin secretion is an ATP-dependent process, the 
Table I. Characteristics and properties of the sirtuins family.

\begin{tabular}{|c|c|c|c|c|c|}
\hline Name & Class & Length, aa & Location of catalytic domain, aa & Primary location & Catalytic activities \\
\hline SIRT1 & I & 747 & $244-498$ & Nucleus & $\begin{array}{l}\text { Deacetylation } \\
\text { Deacylation }\end{array}$ \\
\hline SIRT2 & I & 389 & $65-340$ & Cytoplasm & $\begin{array}{l}\text { Deacetylation } \\
\text { Deacylation } \\
\text { Demyristoylation }\end{array}$ \\
\hline SIRT3 & $\mathrm{I}$ & 399 & $126-382$ & Mitochondria & $\begin{array}{l}\text { Deacetylation } \\
\text { Decrotonylation }\end{array}$ \\
\hline SIRT4 & II & 314 & $45-314$ & Mitochondria & $\begin{array}{l}\text { ADP-ribosylation } \\
\text { Deacetylation } \\
\text { Deacylation } \\
\text { Delipoylation }\end{array}$ \\
\hline SIRT5 & III & 310 & 41-309 & Mitochondria & $\begin{array}{l}\text { Deacetylation } \\
\text { Demalonylation } \\
\text { Desuccinylation }\end{array}$ \\
\hline SIRT6 & IV & 355 & $35-274$ & Nucleus & $\begin{array}{l}\text { Deacetylation } \\
\text { ADP-ribosylation } \\
\text { Demyristoylation }\end{array}$ \\
\hline SIRT7 & IV & 400 & $90-331$ & Nucleus & $\begin{array}{l}\text { Deacetylation } \\
\text { Desuccinylation }\end{array}$ \\
\hline
\end{tabular}

inhibition of GDH will lead to a decrease in ATP production in the mitochondria $(36,44)$. Glucose and amino acid metabolism lead to an increased ATP/ADP ratio. Increased levels of ATP can close ATP-sensitive potassium channels which results in the depolarization of the plasma membrane, thus opening voltage-gated L-type calcium channels, facilitating the fusion of insulin-containing secretory vesicles to the plasma membrane to cause insulin exocytosis $(45,46)$. SIRT4 inhibits the metabolism of glutamate, resulting in decreased production of ATP in mitochondria and inhibition of insulin secretion in pancreatic $\beta$ cells (20). In addition, SIRT4 can interact with insulin-degrading enzyme (IDE) and ADP/ATP translocase 2/3 (ANT2/3) to synergistically inhibit insulin secretion (36).

$\mathrm{NAD}^{+}$-dependent deacetylase and lipidmetabolism. Reversible acetylation of lysine residues is an important post-translational modification, which changes the charge of lysine residues and potentially alters enzyme activity, structure, specificity, and subcellular localization of the protein (47). Deacetylation is the most important and common activity of sirtuins. They catalyze deacetylation by breaking the bonds between $\mathrm{NAD}^{+}$ and niacinamide (NAM) ribosomes, transferring the acetylated groups from proteins to ADP-ribose, then releasing the deacetylated products and NAM. 2-O-acetyl-ADP-ribose is generated as a result of the transfer of the acetyl group onto the ADP-ribose residue. NAM is an inhibitor of deacetylation, which can also reverse the reaction to reproduce $\operatorname{NAD}^{+}(25,48)$.

As a member of the sirtuin family, SIRT4 also possesses typical $\mathrm{NAD}^{+}$-dependent deacetylation activity, but this activity is weak, has substrate specificity, and is associated with cellular lipid metabolism (49). In 2010, Nasrin et al (50) found that downregulating the expression of SIRT4, using adenoviral shRNA, in hepatocytes and myocytes significantly enhanced the expression of the genes, which are associated with fatty acid oxidation (FAO), such as MCAD, PDK4, CTP1, PPAR $\alpha$, PGC1 $\alpha$, ERR $\alpha$, and CoxV. Meanwhile, the FAO of hepatocytes and myocytes was also significantly increased. During cellular lipid metabolism, malonyl CoA provides the carbon skeleton for lipogenesis and also inhibits fat oxidation. Therefore, the regulation of malonyl CoA levels can control the balance between lipid anabolism and catabolism. Both acetyl CoA carboxylase (ACC) and malonyl CoA decarboxylase (MCD) regulate the cellular malonyl CoA level. ACC converts acetyl CoA to malonyl CoA, and MCD converts it back to acetyl CoA (51-53). ACC activity is regulated by phosphorylation from the AMP-activated protein kinase (AMPK) signaling pathway (51). MCD activity is regulated via deacetylation by SIRT4. For example, the deacetylation of the K471 residue of MCD by SIRT4 inhibited the activity of MCD, which further repressed the oxidative decomposition of intracellular fatty acids and promoted the synthesis of lipids in white adipose tissue (52). In vivo, SIRT4-knockout mice had a greater exercise tolerance and protection against diet-induced obesity (52).

With the progress of research, it has been found that the regulation of lipid metabolism by SIRT4 involves several mechanisms. Carnitine O-palmitoyltransferase 1, liver isoform (CPT1) is the rate-limiting enzyme to transfer fatty acids into both the inner and outer mitochondrial membranes. The inhibition of SIRT4 by MCD increased the concentration of malonyl CoA, which downregulated CPT1 activity and inhibited fatty acid oxidation (FAO) (53). Peroxisome proliferator-activated receptor- $\alpha(\operatorname{PPAR} \alpha)$ is one of the mediators of the hepatic response to fasting and a ligand-activated transcription factor, which promotes the transcription of genes involved in fatty acid catabolism, such as Lipg, Acot3, Pdk4, Acox1, Cptla and Acadm $(54,55)$. Accompanied by the high expression of SIRT1 
Table II. Enzymatic activities, substrates and metabolic function of SIRT4.

\begin{tabular}{lllr}
\hline Enzymatic activities & \multicolumn{1}{c}{ Substrates } & Metabolic functions & (Refs.) \\
\hline ADP-ribosyltransferase & GDH & $\begin{array}{l}\text { Insulin secretion } \\
\text { Tumor suppressor }\end{array}$ & $(20)$ \\
NAD ${ }^{+}$-dependent deacetylase & MCD & Fatty acids oxidation & $(52,57)$ \\
Lipoamidase & MTP $\alpha$ & Glycolysis, \\
Long-chain deacylase & CH & Cellular metabolism & $(59)$ \\
Lebiotinylase & MCCC & Leucine metabolism, & $(62,66)$ \\
GDH, glutamate dehydrogenase; MCD, malonyl CoA decarboxylase; MTP $\alpha$, mitochondrial trifunctional protein $\alpha$; PDH, pyruvate dehydro- \\
genase; MCCC, methylcrotonyl-CoA carboxylase; ANT2, ADP/ATP translocase 2.
\end{tabular}

both at mRNA and protein levels, SIRT4 decreased the rate of FAO by inhibiting the expression of PPAR $\alpha$ and downstream genes associated with fatty acid catabolism (55). In addition, the deacetylase activity of SIRT4 also acts on the mitochondrial trifunctional protein $\alpha(\mathrm{MTP} \alpha)$ in hepatocytes, which is a critical enzyme for fatty acid $\beta$-oxidation (56). Deacetylation by SIRT4 increased the activity of MTP- $\alpha$, which inhibited the oxidation of fatty acids and eventually led to hepatic steatosis. In contrast, low expression of SIRT4 promoted the acetylation of MTP- $\alpha$, increased cellular FAO, and prevented the development of non-alcoholic fatty liver disease. In addition, SIRT4 might inhibit the activity of enoyl-CoA hydratase $\alpha$-subunit (ECHA), which is an enzyme involved in branched-chain amino acid (BCAA) catabolism, to suppress FAO (57).

Lipoamidase and glycolysis. The pyruvate dehydrogenase (PDH) complex is a mitochondrial complex, and is the rate-limiting enzyme that catalyzes the decarboxylation of pyruvate to produce acetyl CoA. It also links glycolysis to the TCA cycle and consists of three catalytic subunits: E1, a pyruvate decarboxylase; E2, a dihydrolipoyllysine acetyltransferase (DLAT), and E3, a dihydrolipoyl dehydrogenase (58). Mathias et al (59) demonstrated that SIRT4 had a higher NAD ${ }^{+}$-dependent lipoamidase activity compared with the catalytic efficiency for deacetylation, and exhibited a greater enzymatic activity for lipoyl- and biotinyl-lysine modifications. The E2 component of the PDH is a biological substrate of SIRT4 lipoamidase activity. SIRT4 enzymatically hydrolyzes lipoamide cofactors from DLAT, which results in a reduction in PDH lipoyl levels and inhibition of its function (59). PDH controls pyruvate decarboxylation, fueling multiple downstream pathways, such as aerobic oxidation of glucose to the tricarboxylic acid cycle and oxidative phosphorylation (58). These findings suggest that SIRT4 is a critical modulator of mitochondrial function and cellular metabolism by regulating glycolysis via the inhibition of $\mathrm{PDH}$.

Long-chain deacylase and leucine metabolism, ATP homeostasis. Leucine is a BCAA and an effective stimulator of insulin secretion, as it can allosterically activate GDH, thereby promoting glutamine metabolism and providing energy (60). 3-Methylglutaconyl (MGc)-CoA, 3-methylglutaryl (MG)-CoA, and 3-hydroxy-3-methylglutaryl (HMG)-CoA are intermediates of leucine catabolism catalyzed by methylcrotonyl-CoA carboxylase (MCCC) $(60,61)$. MGc-CoA, MG-CoA, and HMG-CoA function as reactive acyl species to covalently modify and inhibit MCCC using a negative feedback loop. The highly conserved $\alpha$-helical region of SIRT4 removes this modification and reduces insulin secretion by promoting leucine catabolism (62). This effect on insulin secretion in SIRT4-knock-out mice led to elevated glucose- and leucine-stimulated basal insulin levels, which resulted in the development of accelerated age-induced glucose intolerance and insulin resistance. Notably, this is varied with genetic backgrounds, SIRT4KO mice on a C57BL/6NJ genetic background have elevated leucine-stimulated insulin levels; however, SIRT4KO mice on a C57BL/6J background do not $(62,63)$. The repression of SIRT4 activity promoted insulin secretion partly via GDH activation. On the other hand, the loss of SIRT4 function resulted in the accumulation of acyl modified MCCC, which repressed leucine catabolism and allowed for leucine to function as an allosteric activator of GDH. In general, SIRT4 regulates insulin secretion in pancreatic $\beta$ cells through ADP-ribosylation of GDH and deacylation of MCCC in a coordinated way $(20,64)$.

The deacyl function of SIRT4 plays a crucial role in ATP homeostasis. ANT2 is a transmembrane protein located in the inner mitochondrial membrane, and the acylation of ANT2 is known to uncouple mitochondria and reduce the efficiency of oxidative phosphorylation (65). SIRT4 regulates oxygen consumption via modulating the coupling efficiency in an ANT2-dependent manner. In the absence of SIRT4, ANT2-dependent uncoupling led to a decrease in cellular ATP levels and activation of a feedback loop, which involved a reverse signaling response from the mitochondria to the nucleus through AMPK, CPT1, PGC1 $\alpha$, and ACC (66).

\section{Functions of SIRT4 in human cancer}

Metabolic reprogramming is an important aspect of cancer cell metabolism, in which glucose and glutamine metabolic reprogramming are two primary forms. In 1927, Warburg et al (67) found that the rate of glycolysis was much faster compared with that in normal cells, even in the presence of sufficient 


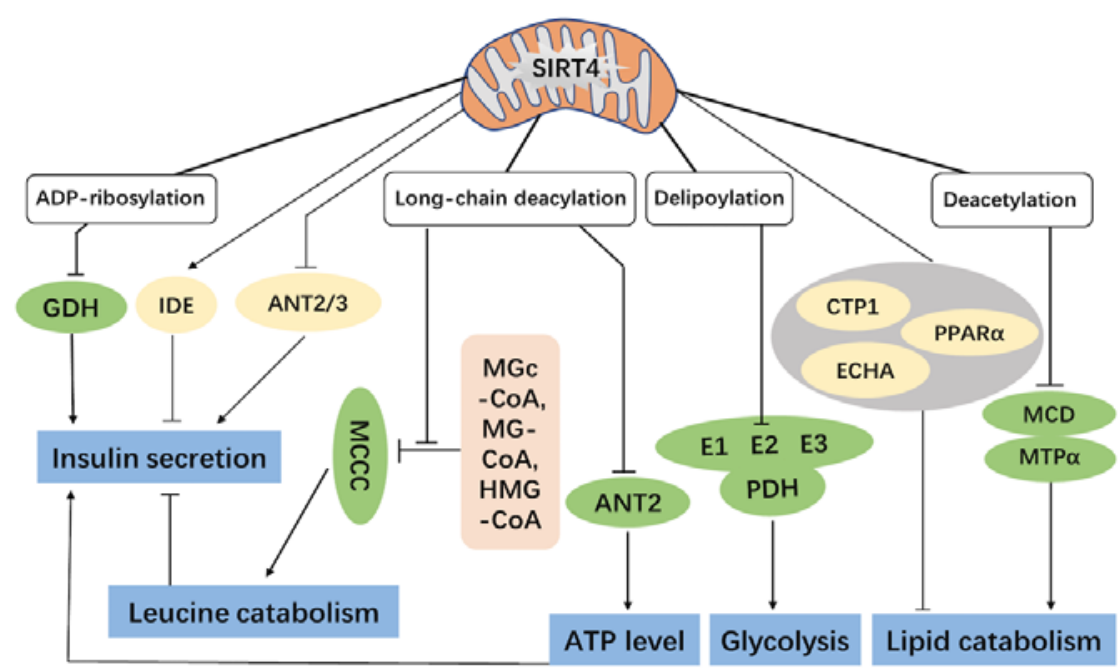

Figure 1. The functions of mitochondrial SIRT4 in cellular metabolism. The ADP-ribosylation on GDH and interactions with IDE and ANT2/3 can regulate insulin secretion in pancreatic $\beta$ cells. The long-chain deacylation on MCCC can regulate leucine catabolism and affect insulin secretion indirectly. The delipoylation on the E2 component of the PDH complex further impacts cellular energy metabolism. The NAD+-dependent deacetylation on MCD and MTP $\alpha$ can inhibit fatty acid $\beta$-oxidation. In addition, SIRT4 also modulates some proteins associated with lipid metabolism (CTP1, PPAR $\alpha$ and ECHA) and finally represses lipid catabolism collectively. GDH, glutamate dehydrogenase; IDE, insulin degrading enzyme; ANT2/3, ADP/ATP translocase 2/3; MCCC, methylcrotonyl-CoA carboxylase; PDH, pyruvate dehydrogenase; MCD, malonyl CoA decarboxylase; MTP $\alpha$, mitochondrial trifunctional protein $\alpha$; MGc, 3-methylglutaconyl; MG, 3-methylglutaryl; HMG, 3-hydroxy-3-methylglutaryl.

oxygen. This phenomenon of aerobic glycolysis, to achieve rapid cell proliferation and energy supply, was termed the Warburg Effect. Glutamine is the most abundant non-essential amino acid in cell plasma (68). Studies have found that the majority of cancer cells can utilize glutamine at a high rate, and some types of cancer cells, such as breast cancer cells, HeLa cervical carcinoma cells and hepatocellular cells cannot survive without an exogenous glutamine supply (69). Glutamine metabolic reprogramming in tumor cells primarily occurs by increased glutamine uptake and glutamine catabolism $(68,70)$. In proliferating cells, the Krebs cycle metabolite, citrate is exported to the cytoplasm for the generation of acetyl CoA. Glutamine serves as an important factor for the replenishment of TCA intermediates which are required due to the continual loss of citrate. In addition, it can also promote the production of intracellular NADH and glutathione to stabilize the intracellular redox balance and regulate the activity of some signal transduction systems, including mTORC1 signaling, the ERK pathway and signaling associated with regulation of mitochondrial ROS production (71-73).

Every member of the sirtuin family has been associated with tumorigenesis (74). SIRT4 serves as a tumor suppressor as it inhibits glutamine metabolism in the mitochondria of cancer cells. Early studies found that SIRT4 mRNA levels were reduced in several malignant tumor tissues, such as bladder cancer, T-cell leukemia, lung cancer, ovarian cancer, and thyroid cancer (75-77). In 2013, Jeong et al (78) discovered that SIRT4 acted as a tumor suppressor by regulating glutamine metabolism in HepG2 cells and PC3 human prostate cancer cells, and SIRT4 knock-out mice spontaneously developed lung cancer. As a result, the tumor suppressor function of SIRT4 had been confirmed in a variety of malignant tumors (Table III). In these studies, the expression level of SIRT4 in normal tissues was significantly higher compared with that in corresponding tumors in the majority of the cancers, and SIRT4 suppressed the biological function of tumor cells in vitro. In addition, several studies have shown that the low expression levels of SIRT4 was significantly associated with the advanced stage of cancer and the poor prognosis of patients (79-95).

In terms of the molecular mechanism, SIRT4 notably represses the metabolism of glutamine through the ADP-ribosylation of GDH, which decreases the energy and material supply required for nucleic acid and protein synthesis to the rapidly proliferating tumor cells (78). This has been confirmed in breast cancer, colorectal cancer, esophageal squamous cell carcinoma, myc-induced B cell lymphoma and thyroid cancer $(78,88,92-94)$. Furthermore, stress-induced DNA damage causes genomic instability in carcinogenic genes, such as TP53, ATM and CDKN2A, which induces SIRT4 expression to inhibit glutamine metabolism, and results in cell cycle arrest (96). This provides sufficient time for DNA damage repair (DDR) and protects the stability of the genome in HeLa cells (97). SIRT4 enhances E-cadherin expression and inhibits the expression of $\mathrm{N}$-cadherin and vimentin, thus inhibiting the process of epithelial-mesenchymal transition, and decreasing the migratory and invasive abilities of gastric and colorectal cancer cells $(84,88)$. In addition, aerobic glycolysis leads to the accumulation of intracellular acidic metabolites, such as pyruvate and lactic acid, and ammonia produced by glutamine metabolism can alleviate this $\mathrm{pH}$ imbalance to maintain the homeostasis of the intracellular environment. However, SIRT4 disturbs this $\mathrm{pH}$ balance and produces an acidic environment in breast cancer (98). This regulatory effect of SIRT4 was modulated by C-terminal binding protein and varied with glucose metabolic levels $(98,99)$. Furthermore, overexpression of SIRT4 blocks cell cycle progression and decreases cancer cell replication by inactivating ERK, p-ERK, cyclin $\mathrm{D}$, and cyclin $\mathrm{E}$ in thyroid cancer and gastric cancer cells $(87,94)$. 
Table III. Summary of research on tumor suppressor function of SIRT4.

\begin{tabular}{|c|c|c|c|c|c|}
\hline Neoplasms & $\begin{array}{l}\text { Expression of } \\
\text { SIRT4 in the } \\
\text { cancer tissues }^{\mathrm{a}}\end{array}$ & $\begin{array}{l}\text { Role of SIRT4 } \\
\text { in the cancer } \\
\text { cell in vitro }\end{array}$ & $\begin{array}{l}\text { Association of expression with } \\
\text { advanced stage and metastasis }\end{array}$ & $\begin{array}{c}\text { Association between } \\
\text { expression level and overall } \\
\text { survival time } \\
\text { a,b }\end{array}$ & (Refs.) \\
\hline HNSCC & Low & NA & NA & Negative correlation & (79) \\
\hline $\mathrm{HCC}$ & Low & Inhibition & Yes & Negative correlation & $(80)$ \\
\hline NSCLC & Low & Inhibition & Yes & Negative correlation & $(81)$ \\
\hline EAC & Low & NA & Yes & NA & $(82)$ \\
\hline NB & Low & Inhibition & Yes & Negative correlation & (83) \\
\hline $\mathrm{GC}$ & Low & Inhibition & Yes & Negative correlation & $(84-87)$ \\
\hline $\mathrm{CRC}$ & Low & Inhibition & Yes & Negative correlation & $(88-90)$ \\
\hline IBC & Low & NA & NA & Negative correlation & (91) \\
\hline ESCC & Low & Inhibition & NA & Negative correlation & (92) \\
\hline BCL & NA & Inhibition & NA & NA & (93) \\
\hline $\mathrm{TC}$ & Low & Inhibition & NA & NA & (94) \\
\hline $\mathrm{PC}$ & NA & Inhibition & NA & NA & $(95)$ \\
\hline
\end{tabular}

In addition, mammalian target of rapamycin complex 1 (mTORC1) was associated with the nutritional status and metabolism of cells, and it repressed the protein level of SIRT4 by destabilizing cAMP-dependent transcription factor ATF-4 (CREB2). On the other hand, low mRNA levels of SIRT4 increased the expression of mTORC downstream genes, such as MYC, CCND1, HIF1A and SREBP1. The mutual inhibition between SIRT4 and mTORC1 was important for the proliferation and survival of colon carcinoma cell line DLD1 and prostate cancer cell line DU145 (100,101). In hepatocellular carcinomas, SIRT4 deletion by shRNA of HL7702 cell line increased mTOR signaling by inhibiting AMPK through the regulation of glutamine catabolism and the AMP/LKB1 pathway (80). In non-small cell lung cancer cell lines, SIRT4 reduced mitochondrial fission by interacting with the Fis-1/Drp1 axis and regulated cell invasive abilities by repressing MEK/ERK activity (81). A study also demonstrated that SIRT4 is a substrate of ubiquitin-like with plant homeodomain and ring finger domains 1 (UHRF1) and negatively regulated aerobic glycolysis, tumor proliferation, and metastasis of pancreatic cancer cells (95). A recent study also suggested that SIRT4 overexpression could heighten the sensitivity of ER-positive breast cancer to tamoxifen via inhibiting the interleukin-6/STAT3 pathway (102).

Notably, SIRT4 plays a dual role in tumorigenesis. SIRT4 plays an important role in DDR and protects the stability of the genome, preventing tumorigenic transformation. In the same way, SIRT4 also protects cancer cells against stresses, such as DNA damage and caloric restriction. For example, the knock-out of SIRT4 in HepG2 cells resulted in decreased cell survival and tumor growth following the DNA damage caused by gamma-radiation, and inversely, the overexpression of SIRT4 in HepG2 cells increased drug and radiation resistance (103). Moreover, SIRT4 promotes cancer cell survival by degrading phosphatase and tensin homolog (PTEN) using the lysosome pathway, which is mediated by IDE (104). PTEN is a lipid phosphatase, which inhibits cancer cell survival and proliferation, and the degradation of PTEN accelerates autophagy during nutritional starvation stresses, such as glucose deprivation. During autophagy, autosomes fuse with lysosomes to form autophagic lysosomes $(105,106)$. It is a salvage pathway to produce proteins, lipids, and carbohydrates for cells to survive in unfavorable conditions (107). Therefore, we hypothesize that SIRT4 is a tumor suppressor prior to the development of cancer; however, once cancer has developed, SIRT4 can still exert a tumor suppressor effect by regulating energy metabolism. Therefore, it has a certain protective effect against cancer cells.

A number of studies have found that SIRT4 is associated with several human diseases. In angiotensin II-induced cardiac hypertrophy in mice, SIRT4 inhibited manganese superoxide dismutase activity and promoted the accumulation of reactive oxygen species in cardiomyocytes, which eventually led to cardiac hypertrophy through the activation of the MAPK/ERK pathway $(108,109)$. Furthermore, the overexpression of SIRT4 protein levels can protect against myocardial ischemia-reperfusion injury by decreasing myocardial infarct size, serum creatine phosphokinase levels, and myocardial apoptosis (110). In the central nervous system, glutamate transport is an energy-dependent process. SIRT4 positively regulated ATP production in neural cells by inhibiting GDH. Specifically, it enhanced the function of the sodium/potassium-ATPase, which led to increased glutamate uptake and glutamate transport to maintain the normal functions of neurons. In vivo, SIRT4-knock-out mice have enhanced seizure phenotypes compared with that in wild-type mice following treatment with a potent excitotoxin kainic acid $(111,112)$. With respect to urogenital diseases, a study demonstrated that Leydig cells treated with lipopolysaccharide led to impaired steroidogenesis 
and enhanced cellular apoptosis via suppression of SIRT4 by the activation of JNK (113). In addition, SIRT4 controls energy metabolism and meiotic apparatus during oocyte maturation, and mouse oocytes with overexpressed SIRT4 protein levels are unable to undergo meiosis completely (114). These data suggest that the roles of SIRT4 in human diseases are both important and complex. Future studies are required to identify connections and detailed mechanisms between them.

\section{Conclusion}

Mitochondria are vital for maintaining an energy balance in cellular and organismal physiology, and aberrant mitochondrial function has been associated with cellular dysfunctions and metabolic diseases. Mitochondrial dysfunction diseases are a group of genetically heterogeneous diseases that can involve any organs, onset at any age, and be inherited from an autosome, the $\mathrm{X}$ chromosome, or maternally (115). The sirtuin family is evolutionary conserved and exhibits a wide range of biological functions in life span, gene transcription, cell proliferation, differentiation, apoptosis, genome stability, cellular metabolism, tumorigenesis, energy homeostasis, DNA damage and stress responses (14-17). Among them, SIRT4 is predominately located in mitochondria and regulates its functions through some known and unknown mechanisms. Recent studies have unraveled numerous biological processes and human diseases, which are regulated by SIRT4 $(116,117)$. However, there are still two crucial issues that require further investigation to provide a more comprehensive understanding of SIRT4.

The first problem is that SIRT4 is still the least well-known mammalian sirtuin; no convincing enzymatic activity has been verified for SIRT4, and there are limited studies on SIRT4 modulators. Its key roles in metabolism and several catalytic activities have been reported; however, more structural information is required to uncover the robust activity and investigate the regulatory mechanisms of SIRT4. To address this gap in the knowledge, the development of effective chemical modulators of SIRT4 is important. Then, these modulators could be applied to scientific research to elucidate the detailed functions of SIRT4, and eventually, for use in clinical diagnosis, treatment or prognosis prediction $(7,21,118)$.

The second problem relates to all seven members of the sirtuin family. Biologically, each of them has a complicated regulatory mechanism via acting on substrates or being modulated by upstream proteins. In fact, their functions are not independent of each other, and numerous studies have confirmed that there is a tight interaction between the sirtuins family. For example, the mRNA level of SIRT4 in granulocytes and monocytes of patients with type 2 diabetes was lower, while the mRNA level of SIRT1 was higher, compared with that in healthy individuals; however, the interaction between these two SIRTs remains unknown (119). In addition, SIRT1 plays a complicated and important role in cardiovascular metabolic diseases (23). Furthermore, SIRT4 ribosylated GDH and decreased its activity; however, SIRT3 deacetylated GDH and increased its activity (120). Both SIRT-4 and -6 have anti-inflammatory functions (121), whereas the activities of SIRT-4 and -5 partly overlap, and collectively regulate the metabolism of BCAA (122). Therefore, a valid network exists among the seven sirtuins, in which they are involved in similar pathways. However, their specific mechanisms remain unknown, and in the future, an extensive amount of investigation is required to clarify this complex network.

\section{Acknowledgements}

Not applicable.

\section{Funding}

This study was partly supported by the National Natural Science Foundation of China (grant no. 81672523) and the Shenyang Plan Project of Science and Technology (grant no. 17-230-098).

\section{Availability of data and materials}

Not applicable.

\section{Authors' contributions}

CW drafted the original manuscript. CW and YL created the tables, designed the figures and performed the literature review. YZ was involved in drafting the initial manuscript, read and approved the final manuscript. CK reviewed and revised the manuscript for important intellectual content. All authors read and approved the final manuscript.

\section{Ethics approval and consent to participate}

Not applicable.

\section{Patient consent for publication}

Not applicable.

\section{Competing interests}

The authors declare that they have no competing interests.

\section{References}

1. Wang Y, He J, Liao M, Hu M, Li W, Ouyang H, Wang X, Ye T, Zhang Y and Ouyang L: An overview of Sirtuins as potential therapeutic target: Structure, function and modulators. Eur J Med Chem 161: 48-77, 2019.

2. Jing $\mathrm{H}$ and Lin $\mathrm{H}$ : Sirtuins in epigenetic regulation. Chem Rev 115: 2350-2375, 2015.

3. Hirschey MD: Old enzymes, new tricks: Sirtuins are NAD(+)-dependent de-acylases. Cell Metab 14: 718-719, 2011.

4. Klar AJ, Fogel S and Macleod K: MAR1-a Regulator of the HMa and HMalpha Loci in SACCHAROMYCES CEREVISIAE. Genetics 93: 37-50, 1979.

5. Michan S and Sinclair D: Sirtuins in mammals: Insights into their biological function. Biochem J 404: 1-13, 2007.

6. Frye RA: Phylogenetic classification of prokaryotic and eukaryotic Sir2-like proteins. Biochem Biophys Res Commun 273: 793-798, 2000.

7. Li Y, Zhou Y, Wang F, Chen X, Wang C, Wang J, Liu T, Li Y and He B: SIRT4 is the last puzzle of mitochondrial sirtuins. Bioorg Med Chem 26: 3861-3865, 2018.

8. Tanno M, Sakamoto J, Miura T, Shimamoto K and Horio Y: Nucleocytoplasmic shuttling of the NAD+-dependent histone deacetylase SIRT1. J Biol Chem 282: 6823-6832, 2007.

9. Liszt G, Ford E, Kurtev M and Guarente L: Mouse Sir2 homolog SIRT6 is a nuclear ADP-ribosyltransferase. J Biol Chem 280: 21313-21320, 2005. 
10. Kumar S and Lombard DB: Mitochondrial sirtuins and their relationships with metabolic disease and cancer. Antioxid Redox Signal 22: 1060-1077, 2015.

11. Michishita E, Park JY, Burneskis JM, Barrett JC and Horikawa I: Evolutionarily conserved and nonconserved cellular localizations and functions of human SIRT proteins. Mol Biol Cell 16 4623-4635, 2005.

12. Mei Z, Zhang X, Yi J, Huang J, He J and Tao Y: Sirtuins in metabolism, DNA repair and cancer. J Exp Clin Cancer Res 35: $182,2016$.

13. Vaquero A, Scher MB, Lee DH, Sutton A, Cheng HL, Alt FW, Serrano L, Sternglanz R and Reinberg D: SirT2 is a histone deacetylase with preference for histone $\mathrm{H} 4 \mathrm{Lys} 16$ during mitosis. Genes Dev 20: 1256-1261, 2006.

14. Jeong SM and Haigis MC: Sirtuins in cancer: A balancing act between genome stability and metabolism. Mol Cells 38: $750-758,2015$.

15. German NJ and Haigis MC: Sirtuins and the metabolic hurdles in cancer. Curr Biol 25: R569-R583, 2015.

16. Grabowska W, Sikora E and Bielak-Zmijewska A: Sirtuins, a promising target in slowing down the ageing process. Biogerontology 18: 447-476, 2017.

17. Kupis W, Palyga J, Tomal E and Niewiadomska E: The role of sirtuins in cellular homeostasis. J Physiol Biochem 72: 371-380, 2016.

18. Frye RA: Characterization of five human cDNAs with homology to the yeast SIR2 gene: Sir2-like proteins (sirtuins) metabolize NAD and may have protein ADP-ribosyltransferase activity. Biochem Biophys Res Commun 260: 273-279, 1999.

19. North BJ, Marshall BL, Borra MT, Denu JM and Verdin E: The human Sir2 ortholog, SIRT2, is an NAD+-dependent tubulin deacetylase. Mol Cell 11: 437-444, 2003.

20. Haigis MC, Mostoslavsky R, Haigis KM, Fahie K, Christodoulou DC, Murphy AJ, Valenzuela DM, Yancopoulos GD, Karow M, Blander G, et al: SIRT4 inhibits glutamate dehydrogenase and opposes the effects of calorie restriction in pancreatic beta cells. Cell 126: 941-954, 2006.

21. Kumar S and Lombard DB: For certain, SIRT4 activities! Trends Biochem Sci 42: 499-501, 2017.

22. Shi JX, Wang QJ, Li H and Huang Q: SIRT4 overexpression protects against diabetic nephropathy by inhibiting podocyte apoptosis. Exp Ther Med 13: 342-348, 2017.

23. Kane AE and Sinclair DA: Sirtuins and NAD(+) in the development and treatment of metabolic and cardiovascular diseases. Circ Res 123: 868-885, 2018.

24. Howitz KT, Bitterman KJ, Cohen HY, Lamming DW, Lavu S, Wood JG, Zipkin RE, Chung P, Kisielewski A, Zhang LL, et al: Small molecule activators of sirtuins extend Saccharomyces cerevisiae lifespan. Nature 425: 191-196, 2003.

25. McGuinness D, McGuinness DH, McCaul JA and Shiels PG: Sirtuins, bioageing, and cancer. J Aging Res 2011: 235754, 2011.

26. Onyango P, Celic I, McCaffery JM, Boeke JD and Feinberg AP. SIRT3, a human SIR2 homologue, is an NAD-dependent deacetylase localized to mitochondria. Proc Natl Acad Sci USA 99: 13653-13658, 2002.

27. Schwer B, North BJ, Frye RA, Ott M and Verdin E: The human silent information regulator (Sir)2 homologue hSIRT3 is a mitochondrial nicotinamide adenine dinucleotide-dependent deacetylase. J Cell Biol 158: 647-657, 2002.

28. Finnin MS, Donigian JR and Pavletich NP: Structure of the histone deacetylase SIRT2. Nat Struct Biol 8: 621-625, 2001.

29. Bellamacina CR: The nicotinamide dinucleotide binding motif: A comparison of nucleotide binding proteins. FASEB J 10: 1257-1269, 1996.

30. Sanders BD, Jackson B and Marmorstein R: Structural basis for sirtuin function: What we know and what we don't. Biochim Biophys Acta 1804: 1604-1616, 2010.

31. Moniot S, Weyand M and Steegborn C: Structures, substrates, and regulators of Mammalian sirtuins-opportunities and challenges for drug development. Front Pharmacol 3: 16, 2012.

32. Yuan $\mathrm{H}$ and Marmorstein R: Structural basis for sirtuin activity and inhibition. J Biol Chem 287: 42428-42435, 2012.

33. Moniot S, Schutkowski M and Steegborn C: Crystal structure analysis of human Sirt2 and its ADP-ribose complex. J Struct Biol 182: 136-143, 2013.

34. Zhou Y, Zhang H, He B, Du J, Lin H, Cerione RA and Hao Q: The bicyclic intermediate structure provides insights into the desuccinylation mechanism of human sirtuin 5 (SIRT5). J Biol Chem 287: 28307-28314, 2012.
35. Jin L, Wei W, Jiang Y, Peng H, Cai J, Mao C, Dai H, Choy W, Bemis JE, Jirousek, et al: Crystal structures of human SIRT3 displaying substrate-induced conformational changes. J Biol Chem 284: 24394-24405, 2009.

36. Ahuja N, Schwer B, Carobbio S, Waltregny D, North BJ, Castronovo V, Maechler P and Verdin E: Regulation of insulin secretion by SIRT4, a mitochondrial ADP-ribosyltransferase. J Biol Chem 282: 33583-33592, 2007.

37. Pannek M, Simic Z, Fuszard M, Meleshin M, Rotili D, Mai A, Schutkowski M and Steegborn C: Crystal structures of the mitochondrial deacylase Sirtuin 4 reveal isoform-specific acyl recognition and regulation features. Nat Commun 8: 1513, 2017.

38. Kato Y, Kihara H, Fukui K and Kojima M: A ternary complex model of Sirtuin4-NAD ${ }^{+}$-Glutamate dehydrogenase. Comput Biol Chem 74: 94-104, 2018.

39. Madsen AS, Andersen C, Daoud M, Anderson KA, Laursen JS, Chakladar S, Huynh FK, Colaço AR, Backos DS, Fristrup P, et al: Investigating the sensitivity of NAD+-dependent sirtuin deacylation activities to NADH. J Biol Chem 291: 7128-7141, 2016.

40. Feldman JL, Dittenhafer-Reed KE, Kudo N, Thelen JN, Ito A, Yoshida M and Denu JM: Kinetic and structural basis for Acyl-Group Selectivity and $\mathrm{NAD}(+)$ Dependence in Sirtuin-Catalyzed Deacylation. Biochemistry 54: 3037-3050, 2015.

41. Gorrini C, Harris IS and Mak TW: Modulation of oxidative stress as an anticancer strategy. Nat Rev Drug Discov 12: 931-947, 2013.

42. Fernandez-Marcos PJ and Serrano M: Sirt4: The glutamine gatekeeper. Cancer Cell 23: 427-428, 2013.

43. Altman BJ, Stine ZE and Dang CV: From Krebs to clinic: Glutamine metabolism to cancer therapy. Nat Rev Cancer 16: 749, 2016.

44. Argmann C and Auwerx J: Insulin secretion: SIRT4 gets in on the act. Cell 126: 837-839, 2006.

45. Lang J: Molecular mechanisms and regulation of insulin exocytosis as a paradigm of endocrine secretion. Eur J Biochem 259: 3-17, 1999.

46. Ashcroft FM, Proks P, Smith PA, Ammala C, Bokvist K and Rorsman P: Stimulus-secretion coupling in pancreatic beta cells. J Cell Biochem 55 (Suppl): S54-S65, 1994.

47. Glozak MA, Sengupta N, Zhang X and Seto E: Acetylation and deacetylation of non-histone proteins. Gene 363: 15-23, 2005.

48. Sauve AA, Wolberger C, Schramm VL and Boeke JD: The biochemistry of sirtuins. Annu Rev Biochem 75: 435-465, 2006.

49. Feldman JL, Baeza J and Denu JM: Activation of the protein deacetylase SIRT6 by long-chain fatty acids and widespread deacylation by mammalian sirtuins. J Biol Chem 288: 31350-31356, 2013.

50. Nasrin N, Wu X, Fortier E, Feng Y, Bare' OC, Chen S, Ren X, Wu Z, Streeper RS and Bordone L: SIRT4 regulates fatty acid oxidation and mitochondrial gene expression in liver and muscle cells. J Biol Chem 285: 31995-32002, 2010.

51. Hardie DG: Sensing of energy and nutrients by AMP-activated protein kinase. Am J Clin Nutr 93: 891S-896, 2011.

52. Laurent G,German NJ, Saha AK, de Boer VC,Davies M, Koves TR, Dephoure N, Fischer F, Boanca G, Vaitheesvaran B, et al: SIRT4 coordinates the balance between lipid synthesis and catabolism by repressing malonyl CoA decarboxylase. Mol Cell 50: 686-698, 2013.

53. Saggerson D: Malonyl-CoA, a key signaling molecule in mammalian cells. Annu Rev Nutr 28: 253-272, 2008.

54. Kersten S, Seydoux J, Peters JM, Gonzalez FJ, Desvergne B and Wahli W: Peroxisome proliferator-activated receptor alpha mediates the adaptive response to fasting. J Clin Invest 103: 1489-1498, 1999.

55. Laurent G, de Boer VC, Finley LW, Sweeney M, Lu H, Schug TT, Cen Y, Jeong SM, Li X, Sauve AA and Haigis MC: SIRT4 represses peroxisome proliferator-activated receptor $\alpha$ activity to suppress hepatic fat oxidation. Mol Cell Biol 33: 4552-4561, 2013.

56. Ibdah JA, Paul H, Zhao Y, Binford S, Salleng K, Cline M, Matern D, Bennett MJ, Rinaldo P and Strauss AW: Lack of mitochondrial trifunctional protein in mice causes neonatal hypoglycemia and sudden death. J Clin Invest 107: 1403-1409, 2001.

57. Guo L, Zhou SR, Wei XB, Liu Y, Chang XX, Liu Y, Ge X, Dou X, Huang HY, Qian SW, et al: Acetylation of mitochondrial trifunctional protein $\alpha$-subunit enhances its stability to promote fatty acid oxidation and is decreased in nonalcoholic fatty liver disease. Mol Cell Biol 36: 2553-2567, 2016. 
58. Zhou ZH, McCarthy DB, O'Connor CM, Reed LJ and Stoops JK The remarkable structural and functional organization of the eukaryotic pyruvate dehydrogenase complexes. Proc Natl Acad Sci USA 98: 14802-14807, 2001.

59. Mathias RA, Greco TM, Oberstein A, Budayeva HG, Chakrabarti R, Rowland EA, Kang Y, Shenk T and Cristea IM: Sirtuin 4 is a lipoamidase regulating pyruvate dehydrogenase complex activity. Cell 159: 1615-1625, 2014.

60. MacDonald MJ, Fahien LA, Brown LJ, Hasan NM, Buss JD and Kendrick MA: Perspective: Emerging evidence for signaling roles of mitochondrial anaplerotic products in insulin secretion. Am J Physiol Endocrinol Metab 288: E1-E15, 2005.

61. Sener A and Malaisse WJ: L-leucine and a nonmetabolized analogue activate pancreatic islet glutamate dehydrogenase. Nature 288: 187-189, 1980.

62. Anderson KA, Huynh FK, Fisher-Wellman K, Stuart JD, Peterson BS, Douros JD, Wagner GR, Thompson JW, Madsen AS Green MF, et al: SIRT4 is a lysine deacylase that controls leucine metabolism and insulin secretion. Cell Metab 25: 838-855 e15, 2017.

63. Huynh FK, Hu X, Lin Z, Johnson JD and Hirschey MD: Loss of sirtuin 4 leads to elevated glucose- and leucine-stimulated insulin levels and accelerated age-induced insulin resistance in multiple murine genetic backgrounds. J Inherit Metab Dis 41: 59-72, 2018.

64. Zaganjor E, Vyas S and Haigis MC: SIRT4 is a regulator of insulin secretion. Cell Chem Biol 24: 656-658, 2017

65. Klingenberg M: The ADP and ATP transport in mitochondria and its carrier. Biochim Biophys Acta 1778: 1978-2021, 2008

66. Ho L, Titus AS, Banerjee KK, George S, Lin W, Deota S, Saha AK, Nakamura K, Gut P, Verdin E and Kolthur-Seetharam U: SIRT4 regulates ATP homeostasis and mediates a retrograde signaling via AMPK. Aging (Albany NY) 5: 835-849, 2013.

67. Warburg $\mathrm{O}$, Wind $\mathrm{F}$ and Negelein $\mathrm{E}$ : The metabolism of tumors in the body. J Gen Physiol 8: 519-530, 1927.

68. Vander Heiden MG, Lunt SY, Dayton TL, Fiske BP, Israelsen WJ, Mattaini KR, Vokes NI, Stephanopoulos G, Cantley LC, Metallo CM and Locasale JW: Metabolic pathway alterations that support cell proliferation. Cold Spring Harb Symp Quant Biol 76: 325-334, 2011.

69. Fuchs BC and Bode BP: Stressing out over survival: Glutamine as an apoptotic modulator. J Surg Res 131: 26-40, 2006.

70. Wise DR, DeBerardinis RJ, Mancuso A, Sayed N, Zhang XY, Pfeiffer HK, Nissim I, Daikhin E, Yudkoff M, McMahon SB and Thompson CB: Myc regulates a transcriptional program that stimulates mitochondrial glutaminolysis and leads to glutamine addiction. Proc Natl Acad Sci USA 105: 18782-18787, 2008 .

71. DeBerardinis RJ, Mancuso A, Daikhin E, Nissim I, Yudkoff M, Wehrli S and Thompson CB: Beyond aerobic glycolysis: Transformed cells can engage in glutamine metabolism that exceeds the requirement for protein and nucleotide synthesis Proc Natl Acad Sci USA 104: 19345-19350, 2007.

72. Daye D and Wellen KE: Metabolic reprogramming in cancer: Unraveling the role of glutamine in tumorigenesis. Semin Cell Dev Biol 23: 362-369, 2012.

73. Lukey MJ, Wilson KF and Cerione RA: Therapeutic strategies impacting cancer cell glutamine metabolism. Future Med Chem 5: 1685-1700, 2013

74. Yuan H, Su L and Chen WY: The emerging and diverse roles of sirtuins in cancer: A clinical perspective. Onco Targets Ther 6: 1399-1416, 2013.

75. Garber ME, Troyanskaya OG, Schluens K, Petersen S, Thaesler Z, Pacyna-Gengelbach M, van de Rijn M, Rosen GD, Perou CM, Whyte RI, et al: Diversity of gene expression in adenocarcinoma of the lung. Proc Natl Acad Sci USA 98: 13784-13789, 2001

76. Blaveri E, Simko JP, Korkola JE, Brewer JL, Baehner F, Mehta K, Devries S, Koppie T, Pejavar S, Carroll P and Waldman FM: Bladder cancer outcome and subtype classification by gene expression. Clin Cancer Res 11: 4044-4055, 2005.

77. Choi YL, Tsukasaki K, O'Neill MC, Yamada Y, Onimaru Y, Matsumoto K, Ohashi J, Yamashita Y, Tsutsumi S, Kaneda R, et al: A genomic analysis of adult T-cell leukemia Oncogene 26: 1245-1255, 2007.

78. Jeong SM, Xiao C, Finley LW, Lahusen T, Souza AL, Pierce K, Li YH, Wang X, Laurent G, German NJ, et al: SIRT4 has tumor-suppressive activity and regulates the cellular metabolic response to DNA damage by inhibiting mitochondrial glutamine metabolism. Cancer Cell 23: 450-463, 2013.
79. Mahjabeen I and Kayani MA: Loss of mitochondrial tumor suppressor genes expression is associated with unfavorable clinical outcome in head and neck squamous cell carcinoma: Data from retrospective study. PLoS One 11: e0146948, 2016.

80. Wang YS, Du L, Liang X, Meng P, Bi L, Wang YL, Wang C and Tang B: Sirtuin 4 depletion promotes hepatocellular carcinoma tumorigenesis through regulating adenosine-monophosphate-activated protein kinase alpha/mammalian target of rapamycin axis in mice. Hepatology 69: 1614-1631, 2019.

81. Fu L, Dong Q, He J, Wang X, Xing J, Wang E, Qiu X and Li Q: SIRT4 inhibits malignancy progression of NSCLCs, through mitochondrial dynamics mediated by the ERK-Drp1 pathway. Oncogene 36: 2724-2736, 2017.

82. Chen X, Lai X, Wu C, Tian Q, Lei T, Pan J and Huang G: Decreased SIRT4 protein levels in endometrioid adenocarcinoma tissues are associated with advanced AJCC stage. Cancer Biomark 19: 419-424, 2017.

83. Wang Y, Guo Y, Gao J and Yuan X: Tumor-suppressive function of SIRT4 in neuroblastoma through mitochondrial damage. Cancer Manag Res 10: 5591-5603, 2018

84. Sun H, Huang D, Liu G, Jian F, Zhu J and Zhang L: SIRT4 acts as a tumor suppressor in gastric cancer by inhibiting cell proliferation, migration, and invasion. Onco Targets Ther 11: 3959-3968, 2018.

85. Huang G, Cui F, Yu F, Lu H, Zhang M, Tang H and Peng Z Sirtuin-4 (SIRT4) is downregulated and associated with some clinicopathological features in gastric adenocarcinoma. Biomed Pharmacother 72: 135-139, 2015.

86. Shen X, Li P, Xu Y, Chen X, Sun H, Zhao Y, Liu M and Zhang W: Association of sirtuins with clinicopathological parameters and overall survival in gastric cancer. Oncotarget 8: 74359-74370, 2017.

87. Hu Y, Lin J, Lin Y, Chen X, Zhu G and Huang G: Overexpression of SIRT4 inhibits the proliferation of gastric cancer cells through cell cycle arrest. Oncol Lett 17: 2171-2176, 2019.

88. Miyo M, Yamamoto H, Konno M, Colvin H, Nishida N, Koseki J, Kawamoto K, Ogawa H, Hamabe A, Uemura M, et al: Tumour-suppressive function of SIRT4 in human colorectal cancer. Br J Cancer 113: 492-499, 2015.

89. Huang G, Cheng J, Yu F, Liu X, Yuan C, Liu C, Chen X and Peng Z: Clinical and therapeutic significance of sirtuin-4 expression in colorectal cancer. Oncol Rep 35: 2801-2810, 2016.

90. Zhu Y, Wang G, Li X, Wang T, Weng M and Zhang Y: Knockout of SIRT4 decreases chemosensitivity to 5-FU in colorectal cancer cells. Oncol Lett 16: 1675-1681, 2018.

91. Shi Q, Liu T, Zhang X, Geng J, He X, Nu M and Pang D: Decreased sirtuin 4 expression is associated with poor prognosis in patients with invasive breast cancer. Oncol Lett 12: 2606-2612, 2016.

92. Nakahara Y, Yamasaki M, Sawada G, Miyazaki Y, Makino T, Takahashi T, Kurokawa Y, Nakajima K, Takiguchi S, Mimori K, et al: Downregulation of SIRT4 expression is associated with poor prognosis in esophageal squamous cell carcinoma. Oncology 90: 347-355, 2016.

93. Jeong SM, Lee A, Lee J and Haigis MC: SIRT4 protein suppresses tumor formation in genetic models of Myc-induced B cell lymphoma. J Biol Chem 289: 4135-4144, 2014.

94. Chen Z, Lin J, Feng S, Chen X, Huang H, Wang C, Yu Y, He Y, Han S, Zheng L and Huang G: SIRT4 inhibits the proliferation, migration, and invasion abilities of thyroid cancer cells by inhibiting glutamine metabolism. Onco Targets Ther 12: 2397-2408, 2019.

95. Hu Q, Qin Y, Ji S, Xu W, Liu W, Sun Q, Zhang Z, Liu M, Ni Q, Yu X and Xu X: UHRF1 promotes aerobic glycolysis and proliferation via suppression of SIRT4 in pancreatic cancer. Cancer Lett 452: 226-236, 2019.

96. Negrini S, Gorgoulis VG and Halazonetis TD: Genomic instability-an evolving hallmark of cancer. Nat Rev Mol Cell Biol 11: 220-228, 2010

97. Colombo SL, Palacios-Callender M, Frakich N, Carcamo S, Kovacs I, Tudzarova S and Moncada S: Molecular basis for the differential use of glucose and glutamine in cell proliferation as revealed by synchronized HeLa cells. Proc Natl Acad Sci USA 108: 21069-21074, 2011.

98. Wang L, Zhou H, Wang Y, Cui G and Di LJ: CtBP maintains cancer cell growth and metabolic homeostasis via regulating SIRT4. Cell Death Dis 6: e1620, 2015.

99. Wang L, Li JJ, Guo LY, Li P, Zhao Z, Zhou H and Di LJ: Molecular link between glucose and glutamine consumption in cancer cells mediated by CtBP and SIRT4. Oncogenesis 7: 26, 2018. 
100. Csibi A, Fendt SM, Li C, Poulogiannis G, Choo AY, Chapski DJ, Jeong SM, Dempsey JM, Parkhitko A, Morrison T, et al: The mTORC1 pathway stimulates glutamine metabolism and cell proliferation by repressing SIRT4. Cell 153: 840-854, 2013.

101. van de Ven RAH, Santos D and Haigis MC: Mitochondrial Sirtuins and molecular mechanisms of aging. Trends Mol Med 23: 320-331, 2017.

102. Xing J, Li J, Fu L, Gai J, Guan J and Li Q: SIRT4 enhances the sensitivity of ER-positive breast cancer to tamoxifen by inhibiting the IL-6/STAT3 signal pathway. Cancer Med 8: 7086-7097, 2019.

103. Jeong SM, Hwang S and Seong RH: SIRT4 regulates cancer cell survival and growth after stress. Biochem Biophys Res Commun 470: 251-256, 2016.

104. Liu M, Wang Z, Ren M, Yang X, Liu B, Qi H, Yu M, Song S, Chen S, Liu L, et al: SIRT4 regulates PTEN stability through IDE in response to cellular stresses. FASEB J 33: 5535-5547, 2019.

105. Lee JO, Yang H, Georgescu MM, Di Cristofano A, Maehama T, Shi Y, Dixon JE, Pandolfi P and Pavletich NP: Crystal structure of the PTEN tumor suppressor: Implications for its phosphoinositide phosphatase activity and membrane association. Cell 99: 323-334, 1999

106. Georgescu MM, Kirsch KH, Kaloudis P, Yang H, Pavletich NP and Hanafusa $\mathrm{H}$ : Stabilization and productive positioning roles of the C2 domain of PTEN tumor suppressor. Cancer Res 60: 7033-7038, 2000.

107. Mizushima N and Klionsky DJ: Protein turnover via autophagy: Implications for metabolism. Annu Rev Nutr 27: 19-40, 2007.

108. Luo YX, Tang X, An XZ, Xie XM, Chen XF, Zhao X, Hao DL, Chen HZ and Liu DP: SIRT4 accelerates Ang II-induced pathological cardiac hypertrophy by inhibiting manganese superoxide dismutase activity. Eur Heart J 38: 1389-1398, 2017.

109. Xiao Y, Zhang X, Fan S, Cui G and Shen Z: MicroRNA-497 inhibits cardiac hypertrophy by targeting Sirt4. PLoS One 11: e0168078, 2016

110. Zeng G, Liu H and Wang H: Amelioration of myocardial ischemia-reperfusion injury by SIRT4 involves mitochondrial protection and reduced apoptosis. Biochem Biophys Res Commun 502: 15-21, 2018.

111. Shih J, Liu L, Mason A, Higashimori H and Donmez G: Loss of SIRT4 decreases GLT-1-dependent glutamate uptake and increases sensitivity to kainic acid. J Neurochem 131: 573-581, 2014.
112. Komlos D, Mann KD, Zhuo Y, Ricupero CL, Hart RP, Liu AY and Firestein BL: Glutamate dehydrogenase 1 and SIRT4 regulate glial development. Glia 61: 394-408, 2013.

113. Ramatchandirin B, Sadasivam M, Kannan A and Prahalathan C: Sirtuin 4 regulates lipopolysaccharide mediated leydig cell dysfunction. J Cell Biochem 117: 904-916, 2016.

114. Zeng J, Jiang M, Wu X, Diao F, Qiu D, Hou X, Wang H, Li L, Li C, Ge J, et al: SIRT4 is essential for metabolic control and meiotic structure during mouse oocyte maturation. Aging Cell 17: e12789, 2018.

115. Nunnari J and Suomalainen A: Mitochondria: In sickness and in health. Cell 148: 1145-1159, 2012.

116. Han Y, Zhou S, Coetzee S and Chen A: SIRT4 and its roles in energy and redox metabolism in health, disease and during exercise. Front Physiol 10: 1006, 2019.

117. Min Z, Gao J and Yu Y: The roles of mitochondrial SIRT4 in cellular metabolism. Front Endocrinol (Lausanne) 9: 783 , 2019.

118. Li S and Zheng W: Mammalian Sirtuins SIRT4 and SIRT7. Prog Mol Biol Transl Sci 154: 147-168, 2018.

119. Song R, Xu W, Chen Y, Li Z, Zeng Y and Fu Y: The expression of Sirtuins 1 and 4 in peripheral blood leukocytes from patients with type 2 diabetes. Eur J Histochem 55: e10, 2011.

120. Kim EA, Yang SJ, Choi SY, Lee WJ and Cho SW: Inhibition of glutamate dehydrogenase and insulin secretion by KHG26377 does not involve ADP-ribosylation by SIRT4 or deacetylation by SIRT3. BMB Rep 45: 458-463, 2012.

121. Lappas M: Anti-inflammatory properties of sirtuin 6 in human umbilical vein endothelial cells. Mediators Inflamm 2012: 597514, 2012.

122. Sebastian C, Satterstrom FK, Haigis MC and Mostoslavsky R: From sirtuin biology to human diseases: An update. J Biol Chem 287: 42444-42452, 2012.

This work is licensed under a Creative Commons Attribution-NonCommercial-NoDerivatives 4.0 International (CC BY-NC-ND 4.0) License. 